\title{
Inhaled corticosteroid use and the incidence of lung cancer in COPD
}

\author{
Samy Suissa ${ }^{1,2}$, Sophie Dell'Aniello ${ }^{1,2}$, Anne V. Gonzalez ${ }^{1,2}$ and Pierre Ernst ${ }^{1,2}$ \\ Affiliations: ${ }^{1}$ Center for Clinical Epidemiology, Lady Davis Institute - Jewish General Hospital, Montreal, QC, \\ Canada. ${ }^{2}$ Depts of Epidemiology and Biostatistics and of Medicine, McGill University, Montreal, QC, Canada. \\ Correspondence: Samy Suissa, Centre for Clinical Epidemiology, Jewish General Hospital, 3755 Cote \\ Ste-Catherine, Montreal, Québec, Canada H3 T 1E2. E-mail: samy.suissađmcgill.ca
}

\section{@ERSpublications}

Large cohort study finds no reduction in lung cancer incidence with inhaled corticosteroids in COPD. Other observational studies reporting significant reductions probably affected by time-related biases and confounding from the inclusion of asthma patients. http://bit.ly/34Qeetd

Cite this article as: Suissa S, Dell'Aniello S, Gonzalez AV, et al. Inhaled corticosteroid use and the incidence of lung cancer in COPD. Eur Respir J 2020; 55: 1901720 [https://doi.org/10.1183/ 13993003.01720-2019].

\section{ABSTRACT}

Background: Inhaled corticosteroids (ICS) are suggested for potential chemoprevention of lung cancer. Several observational studies in patients with chronic obstructive pulmonary disease (COPD) reported inconsistent results, either significant reductions in lung cancer incidence with ICS use or no effect. We assessed this association, using an approach that avoided biases affecting some of the studies.

Methods: A cohort of patients with COPD, new users of long-acting bronchodilators over 2000-2014, was formed using the Quebec healthcare databases, and followed until 2015 for a first diagnosis of lung cancer. A 1-year delay after cohort entry was used to avoid protopathic bias and a 1-year latency period was included after the initiation of ICS use. A time-dependent Cox regression model was used to estimate the hazard ratio (HR) of lung cancer associated with ICS exposure, adjusted for covariates.

Results: The cohort involved 63276 subjects, including 63\% receiving ICS, with 3743 lung cancers occurring during a mean follow-up of 5 years. The adjusted HR of lung cancer associated with any ICS exposure was 1.01 (95\% CI 0.94-1.08), relative to no ICS use. The HR with longer time ( $>4$ years) since ICS initiation was 0.92 (95\% CI 0.83-1.03), while with higher mean daily ICS dose (>1000 $\mu \mathrm{g}$ fluticasone equivalents) was 1.36 (95\% CI 1.03-1.81).

Conclusions: Inhaled corticosteroid use is not associated with a reduction in lung cancer incidence in patients with COPD. Observational studies reporting such reduction may have been affected by timerelated biases and the inclusion of patients with asthma. The proposition of a randomised trial warrants some caution. 


\section{Introduction}

Chronic obstructive pulmonary disease (COPD) is now the third leading cause of death in the world and is linked to several comorbidities [1-3]. Lung cancer is one such comorbidity, whose incidence is significantly higher in patients with COPD than in the general population [4]. Several factors have been associated with variations in the incidence of lung cancer in patients with COPD [5].

In linking the two diseases, the inflammation associated with lung cancer and its potential reduction with long-term inhaled corticosteroid (ICS) use has been examined. Some observational studies reported that ICS use is associated with a lower incidence of lung cancer [6, 7]. Certain studies also suggest that this reduction may be dose-dependent [8-11]. However, other observational studies did not find an association between ICS use and lung cancer incidence [12-14]. In addition, data from large randomised trials of ICS in COPD found no decreased incidence of lung cancer [15]. Nevertheless, some find the evidence sufficiently compelling to suggest the conduct of a prospective randomised controlled trial [16]. This issue has important implications as ICS are now used extensively in the treatment of COPD, although the appropriateness of such widespread use has been questioned [17].

These discrepancies among observational studies, as well as with randomised trial data, could be due to the introduction of bias, including time-related biases leading to exposure and outcome misclassification. In this paper, we use data from the Canadian Province of Quebec to assess the association between ICS use and lung cancer incidence, using a study design and data analysis techniques that avoid these sources of bias.

\section{Methods}

\section{Sources of data}

The study was conducted using databases from the Régie de l'Assurance Maladie du Québec, the administration that manages the universal health insurance plan for the 8 million residents of the province of Québec. In particular, these comprise information from all hospital discharge summaries in the Province, including information on cancer diagnoses, for which the sensitivity was shown to be high for lung cancer [18]. In addition, they include data on medical services rendered and prescription drugs dispensed to all people aged $\geqslant 65$ years, welfare recipients, and, since 1996, other residents who opt to join the provincial drug plan. These databases have been used previously to study the epidemiology of COPD and the effects of its treatments [19-22].

\section{Cohort definition}

The study cohort was formed by first identifying all subjects with three or more prescriptions for long-acting muscarinic antagonists (LAMA) or long-acting $\beta$-agonists (LABA, not combined with ICS), dispensed in any 1-year period and on at least two different dates, between 2000 and 2014. To ensure a new-user cohort of treated COPD patients, we included only subjects who had no prescription for these drugs in the 1-year period prior to cohort entry, defined by the first of the three prescriptions, necessitating 1-year enrolment prior to cohort entry. Subjects had to be aged $\geqslant 50$ years at cohort entry. All subjects were followed from cohort entry until the first lung cancer diagnosis, death or the end of insurance drug coverage or data availability (December 31,2015). Patients with a diagnosis of lung cancer or a prescription for an ICS in the year prior to cohort entry were excluded.

\section{Outcome classification}

The first lung cancer event occurring during follow-up was identified from primary inpatient and specialist outpatient diagnoses (International Classification of Diseases (ICD)-9 162.X, ICD-10 C33-C34). Protopathic bias is plausible in this context, since early respiratory symptoms of a yet undiagnosed lung cancer can be treated with bronchodilators, so that some new users of a LABA or LAMA may have initiated their treatment for an imminent lung cancer [23]. Thus, all subjects with $<1$ year of follow-up, including those with a lung cancer diagnosis in the first year of follow-up after cohort entry were excluded.

\section{Exposure classification}

All outpatient prescriptions for ICS, including beclometasone, budesonide, triamcinolone, fluticasone, mometasone, ciclesonide and flunisolide, alone or in a combination inhaler, dispensed after cohort entry were identified. The dose of ICS of each prescription was converted to fluticasone equivalents [24].

To avoid immortal time bias, subjects were classified as "unexposed" to ICS prior to their first prescription for ICS and "exposed" subsequently [25]. Moreover, to include a biologically plausible period of the effect of ICS on lung cancer incidence, we defined a 1-year latency period after the date of the first ICS prescription [26]. Thus, all person-time in this latency period was also classified as unexposed to ICS, while only the person-time after this 1-year latency period was classified as exposed. Consequently, lung 
cancer events occurring during this 1-year latency period were classified as unexposed, and only those occurring subsequently were classified as exposed to ICS.

\section{Data analysis}

We estimated the crude rates and rate ratios of lung cancer incidence associated with ICS exposure according to the person-time under exposure. We used time-dependent Cox proportional hazards regression models to estimate the hazard ratio (HR) of lung cancer incidence associated with ICS exposure according to the time-dependent change in ICS exposure during follow-up, adjusted for potential confounders measured at cohort entry. These covariates included age, sex, COPD hospitalisation and exacerbation in the year prior to cohort entry, as well as comorbidity at cohort entry, including cardiovascular and cerebrovascular diseases, diabetes, renal disease, other cancers (not lung), dementia and rheumatoid disease, among others.

To assess the effect of treated time on lung cancer incidence, the time since the first ICS prescription was categorised as $<2,2-4$ and $>4$ years. To assess the effect of ICS dose, the time-dependent mean daily dose was computed by summing the fluticasone dose-equivalents of all ICS prescriptions dispensed from the first prescription to the time point of the risk set defined by the Cox hazards model, divided by the time from first ICS prescription to the risk set time. The mean ICS dose categorised as $<500,500-1000$ and $>1000 \mu \mathrm{g}$ of fluticasone equivalents. In addition, cubic splines were used with the Cox proportional hazards regression model to estimate the HR of lung cancer incidence over time since ICS initiation and by the mean ICS daily dose.

We performed sensitivity analyses to assess the impact of the various time-related biases observed in some of the observational studies to date. Thus, we removed the 1-year protopathic bias criterion, removed the 1 -year latency period and introduced immortal time bias by classifying a patient who received ICS at any

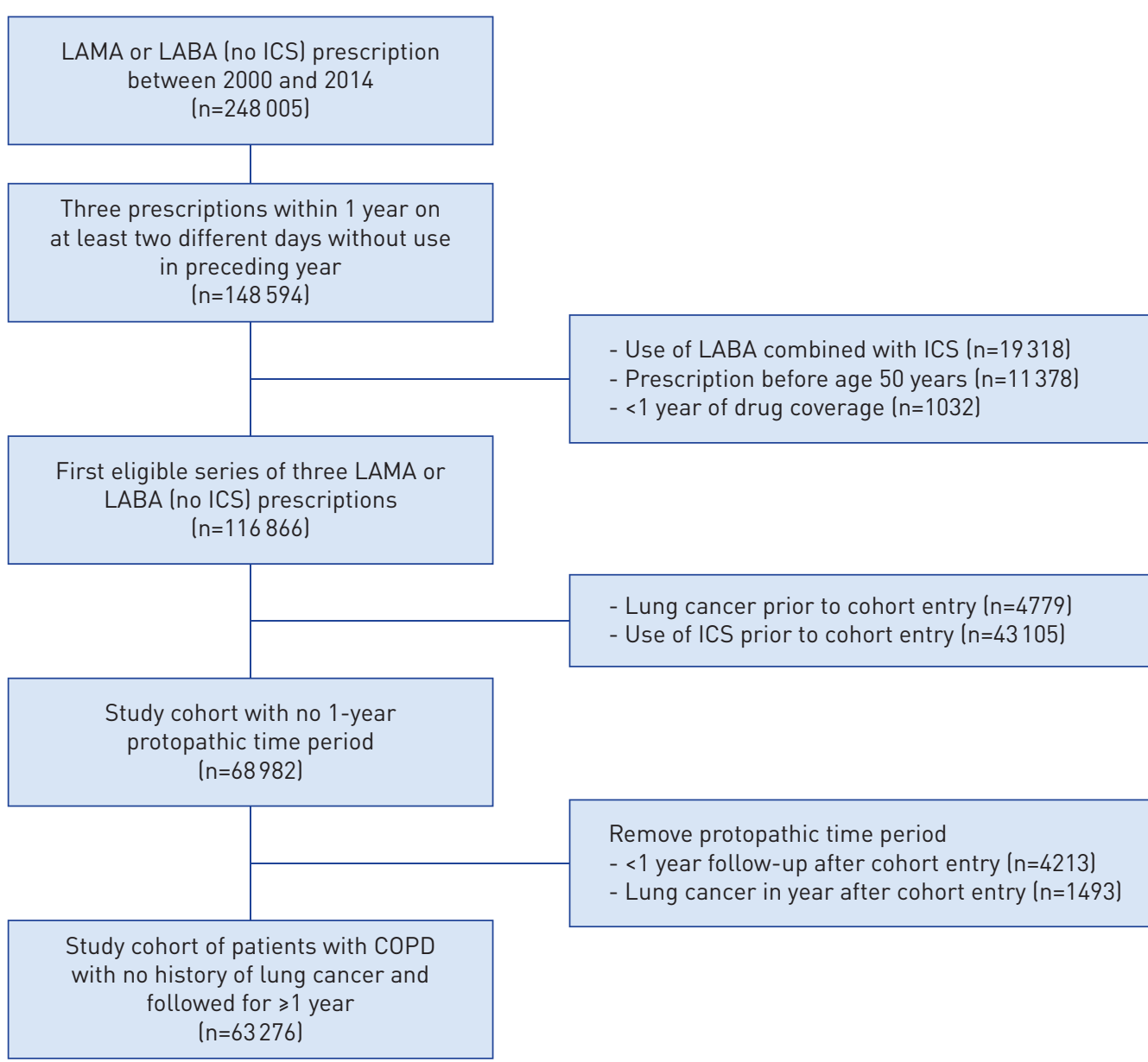

FIGURE 1 Flowchart of the study cohort formation. LAMA: long-acting muscarinic antagonists; LABA: long-acting $\beta$-agonists; ICS: inhaled corticosteroids; COPD: chronic obstructive pulmonary disease. 
time as exposed to ICS as of cohort entry. All analyses were performed using SAS (version 9.4; SAS, Cary, NC, USA). The study was approved by the ethics committee of the Jewish General Hospital (JGH Protocol \#16-225; Montreal, QC, Canada).

\section{Results}

The study cohort initially included 68982 COPD patients who were new users of LABA or LAMA, aged $\geqslant 50$ years, between 2000 and 2014 (figure 1). The mean follow-up was 4.7 years (maximum 16 years), during which 5236 were diagnosed with lung cancer (incidence rate 16.0 per 1000 person-years). A total of 5706 patients, including 1493 lung cancer cases, who had <1 year of follow-up were excluded to avoid protopathic bias. Thus, the final study cohort included 63276 COPD patients of mean age 71 years at cohort entry; $47 \%$ were female.

$40164(63 \%)$ subjects received a prescription for ICS at some point during follow-up. The mean time between cohort entry and the first ICS prescription among the users was 1.2 (range 0-15) years. Table 1 presents the subject characteristics of the study cohort at baseline, by use of ICS during follow-up. In the year prior to cohort entry, 5.5\% had been hospitalised for COPD and 20\% had a COPD exacerbation, defined by a prescription for an oral glucocorticoid or a hospitalisation for COPD.

The overall rate of lung cancer incidence in the final study cohort is 11.5 per 1000 person-years. After removing the 1-year protopathic period and considering the 1-year latency, the adjusted HR of lung cancer incidence with time-dependent ICS use is 1.01 (95\% CI 0.94-1.08) relative to non-use (table 2). There is a trend towards a lower HR with longer time (>4 years) since first ICS prescription (HR 0.92, 95\% CI 0.83 1.03) relative to non-use (table 2 and figure 2). The effect fluctuates with respect to the mean daily ICS dose, as $<500 \mu \mathrm{g}$ fluticasone equivalents is associated with a lower incidence of lung cancer (HR 0.91, 95\%

TABLE 1 Baseline characteristics of study cohort of patients with chronic obstructive pulmonary disease (COPD) initiating treatment with long-acting $\beta$-agonists or long-acting muscarinic antagonists, Quebec, 2000-2014, according to use of inhaled corticosteroids (ICS) during follow-up

\begin{tabular}{|c|c|c|}
\hline & No ICS & Any ICS \\
\hline Patients & 23112 & 40164 \\
\hline Female & $10189(44.1)$ & $19861(49.4)$ \\
\hline Age years & $72.3 \pm 10.1$ & $71.0 \pm 9.7$ \\
\hline \multicolumn{3}{|l|}{ Age group } \\
\hline $50-64$ years & $5246(22.7)$ & $10221(25.4)$ \\
\hline $65-74$ years & $7791(33.7)$ & $14774(36.8)$ \\
\hline $75-84$ years & 7365 (31.9) & $11826(29.4)$ \\
\hline$\geqslant 85$ years & $2710(11.7)$ & $3343(8.3)$ \\
\hline \multicolumn{3}{|c|}{ COPD events in year prior to cohort entry } \\
\hline Hospitalisation & $886(3.8)$ & $2566(6.4)$ \\
\hline \multicolumn{3}{|l|}{ Exacerbations } \\
\hline None & 19719 (85.3) & 31055 (77.3) \\
\hline 1 & $2653(11.5)$ & $7161(17.8)$ \\
\hline$\geqslant 2$ & 740 (3.2) & $1948(4.9)$ \\
\hline \multicolumn{3}{|c|}{ Comorbidity in year prior to cohort entry } \\
\hline Myocardial infarction & $1289(5.6)$ & $1977(4.9)$ \\
\hline Heart failure & $2417(10.5)$ & $3600(9.0)$ \\
\hline Peripheral vascular disease & $1883(8.1)$ & $2828(7.0)$ \\
\hline Cerebrovascular disease & $1041(4.5)$ & $1645(4.1)$ \\
\hline Diabetes & 5359 (23.2) & $8435(21.0)$ \\
\hline Renal disease & $1841(8.0)$ & $2481(6.2)$ \\
\hline Cancer (not lung) & $1379(6.0)$ & $2158(5.4)$ \\
\hline Metastatic cancer & $496(2.1)$ & $699(1.7)$ \\
\hline Dementia & $865(3.7)$ & $1008(2.5)$ \\
\hline Rheumatoid disease & $344(1.5)$ & $559(1.4)$ \\
\hline Peptic ulcer & $187(0.8)$ & $359(0.9)$ \\
\hline Liver disease & $554(2.4)$ & $793(2.0)$ \\
\hline Hemiplegia or paraplegia & $187(0.8)$ & $326(0.8)$ \\
\hline
\end{tabular}


TABLE 2 Crude and adjusted hazard ratios (HRs) of lung cancer incidence with inhaled corticosteroid (ICS) use versus non-use in patients with chronic obstructive pulmonary disease, by time since initiation and by mean daily dose

\begin{tabular}{|c|c|c|c|c|c|}
\hline & $\begin{array}{l}\text { Patients with } \\
\text { events }\end{array}$ & $\begin{array}{l}\text { Person- } \\
\text { years }\end{array}$ & $\begin{array}{l}\text { Rate per } 1000 \\
\text { per year }\end{array}$ & $\begin{array}{l}\text { Crude } \\
\text { HR }\end{array}$ & $\begin{array}{c}\text { Adjusted }^{\#} \text { HR } \\
(95 \% \mathrm{CI})\end{array}$ \\
\hline \multicolumn{6}{|l|}{ Any ICS use } \\
\hline None (reference) & 1645 & 178678 & 9.2 & 1.00 & 1.00 (reference) \\
\hline Any ICS & 2098 & 145396 & 14.4 & 1.02 & $1.01(0.94-1.08)$ \\
\hline \multicolumn{6}{|c|}{ Time since ICS initiation } \\
\hline None (reference) & 1645 & 178678 & 9.2 & 1.00 & 1.00 (reference) \\
\hline $0-2$ years & 503 & 33192 & 15.2 & 1.09 & $1.08(0.97-1.19)$ \\
\hline$>2-4$ years & 726 & 49247 & 14.7 & 1.02 & $1.01(0.92-1.11)$ \\
\hline$>4$ years & 869 & 62956 & 13.8 & 0.93 & $0.92(0.83-1.03)$ \\
\hline \multicolumn{6}{|c|}{$\begin{array}{l}\text { Mean daily dose }{ }^{\pi} \text { since } \\
\text { ICS initiation }\end{array}$} \\
\hline None (reference) & 1645 & 178678 & 9.2 & 1.00 & 1.00 (reference) \\
\hline$>0$ to $<500 \mu \mathrm{g}$ & 1443 & 112030 & 12.9 & 0.91 & $0.91(0.85-0.98)$ \\
\hline 500 to $<1000 \mu \mathrm{g}$ & 604 & 30876 & 19.6 & 1.38 & $1.32(1.20-1.46)$ \\
\hline$\geqslant 1000 \mu \mathrm{g}$ & 51 & 2490 & 20.5 & 1.44 & $1.36(1.03-1.81)$ \\
\hline
\end{tabular}

Data are presented as $n$, unless otherwise stated. ${ }^{\#}$ : after adjusting for covariates in table 1 ; ${ }^{\text {ๆ: dose across }}$ different ICS converted to fluticasone equivalents [24].

CI $0.85-0.98)$, while for $500-1000 \mu \mathrm{g}$ the $\mathrm{HR}$ is 1.32 (95\% CI $1.20-1.46)$ relative to non-use (table 2 and figure 3).

Table 3 presents the sensitivity analyses to assess the impact of protopathic, latency and immortal time biases. When immortal time was properly dealt with by classifying ICS exposure as time-dependent, the effects of ignoring the protopathic and latency periods were found to be somewhat important, resulting in a HR of 1.22 (95\% CI 1.15-1.29), in contrast to the HR 1.01 (95\% CI 0.94-1.08) that accounted for both periods. Conversely, the introduction of immortal time bias that misclassifies the immortal time prior to the first ICS prescription as exposed to ICS instead of unexposed resulted in significant underestimations of the HR, including an estimate of 0.51 (95\% CI 0.48-0.55) even after accounting for 1-year protopathic and latency periods.

\section{Discussion}

Using a large cohort of $>60000$ new users of COPD medications followed for up to 16 years, we found that inhaled corticosteroid use is not associated with a reduction in the incidence of lung cancer. In addition, there was no effect by duration of ICS use, but low doses of ICS were associated with a lower incidence of lung cancer and higher doses with a higher incidence.

The reduction in lung cancer incidence with ICS use was reported by several observational studies, with some suggesting that this reduction may be dose-dependent [6-11]. However, other observational studies

FIGURE 2 Adjusted hazard ratio (95\% Cl) of lung cancer incidence according to time since first inhaled corticosteroid (ICS) prescription, fit by cubic splines. Graph starts at 1 year because of the 1-year latency period.

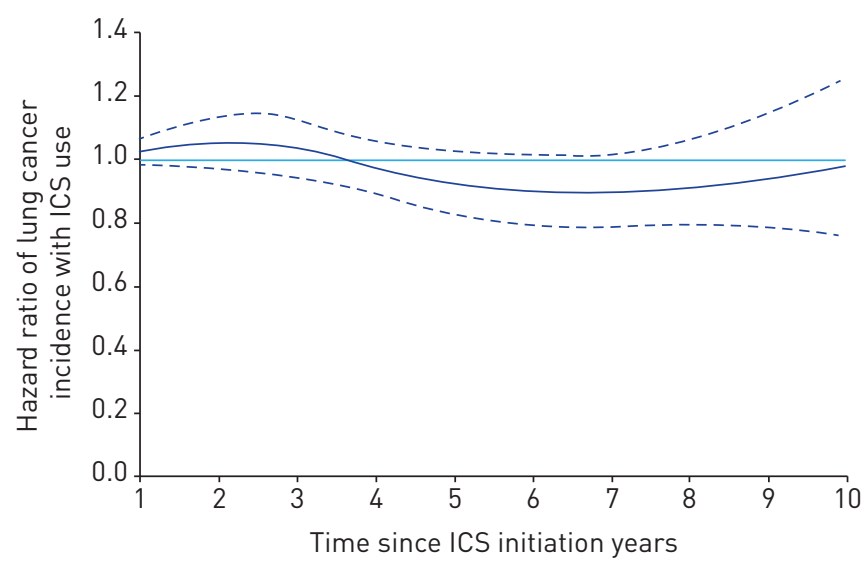


FIGURE 3 Adjusted hazard ratio (95\% Cl) of lung cancer incidence according to mean daily dose lin fluticasone equivalents), fit by cubic splines.

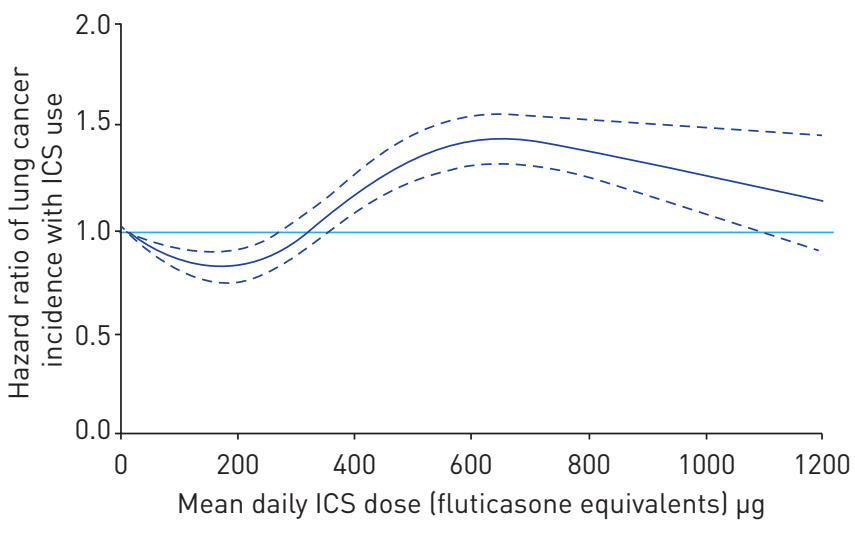

did not find an association [12-14]. Moreover, a review identified randomised trials of ICS in COPD that reported data on lung cancer [15]. None of the trials found a reduction in lung cancer incidence with ICS [27-30]. Furthermore, the Salford COPD randomised trial reported eight lung cancer related deaths in the fluticasone furoate-vilanterol arm compared with two in the usual-care arm, although no data were reported on lung cancer incidence [31].

An important methodological aspect that can explain the reduction in lung cancer incidence found in some of the observational studies is the confounding potential of the "asthma factor", which has affected several studies of ICS effectiveness in COPD [32]. Indeed, patients with asthma are more likely to receive ICS and less likely to develop lung cancer, partly by the differences in smoking prevalence. In some of the

TABLE 3 Sensitivity analyses for the crude and adjusted hazard ratios (HR) of lung cancer incidence with inhaled corticosteroid (ICS) use versus non-use in patients with chronic obstructive pulmonary disease, by the inclusion or exclusion of protopathic and latency time periods, without and with immortal time bias

Patients with events
Person-years

\section{No immortal time bias}

No latency and no protopathic periods None (reference)

Any ICS

No latency and 1-year protopathic period None (reference)

Any ICS

1-year latency and no protopathic period None (reference)

Any ICS

1-year latency and 1-year protopathic period None (reference) Any ICS

\section{With immortal time bias}

No latency and no protopathic periods None (reference) Any ICS

No latency and 1-year protopathic period None (reference) Any ICS

1-year latency and no protopathic period None (reference) Any ICS

1-year latency and 1-year protopathic period None (reference) Any ICS

$\begin{array}{lcccc}2224 & 142493 & 15.6 & 1.00 & 1.00 \text { (reference) } \\ 3012 & 184736 & 16.3 & 1.21 & 1.22 \text { (1.15-1.29) } \\ 1222 & 140288 & 8.7 & 1.00 & 1.00 \text { (reference) } \\ 2521 & 183786 & 13.7 & 1.25 & 1.25 \text { (1.16-1.34) } \\ 3138 & 181833 & 17.3 & 1.00 & 1.00 \text { (reference) } \\ 2098 & 145396 & 14.4 & 1.02 & 1.02 \text { (0.95-1.09) } \\ 1645 & 178678 & 9.2 & 1.00 & 1.00 \text { (reference) } \\ 2098 & 145396 & 14.4 & 1.02 & 1.01 \text { (0.94-1.08) } \\ & & & & \\ 2224 & 92906 & 23.9 & 1.00 & 1.00 \text { (reference) } \\ 3012 & 234322 & 12.9 & 0.55 & 0.54 \text { (0.51-0.57) } \\ 1222 & 90916 & 13.4 & 1.00 & 1.00 \text { (reference) } \\ 2521 & 233158 & 10.8 & 0.72 & 0.70 \text { (0.66-0.76) } \\ 3138 & 107418 & & & \\ 2098 & 219810 & 29.2 & 1.00 & 1.00 \text { (reference) } \\ 1645 & 104264 & 9.5 & 0.33 & 0.32 \text { (0.30-0.34) } \\ 2098 & 219810 & 15.8 & 1.00 & 1.00 \text { (reference) } \\ & & 9.5 & 0.52 & 0.51 \text { (0.48-0.55) }\end{array}$

Data are presented as $n$, unless otherwise stated. ${ }^{*}$ : after adjusting for covariates in table 1. 
studies, a large proportion of the subjects also had a diagnosis of asthma, with minimal or no adjustment for asthma, nor stratification $[6,9,11]$. In one study, the included patients were aged $\geqslant 40$ years with a COPD diagnosis or "self-reported chronic lung disease", but did not report on asthma [8]. Conversely, patients with emphysema who have a higher incidence of lung cancer may be less likely to be treated with ICS because of more severe dyspnoea [33]. Thus, the inability to identify emphysema in the database can also result in a confounded reduction in lung cancer incidence with ICS. Thus, residual confounding by asthma prevalence, asthma severity, emphysema and smoking cannot be ruled out for the significant reductions in lung cancer incidence with ICS use reported in these studies.

Time-related biases are another important methodological issue affecting some of these studies. Our sensitivity analyses demonstrate that immortal time bias can have the greatest impact on these studies. Immortal time bias results from the exposure definition to ICS, for example "based on having filled a prescription after the start of follow-up", which will cause a patient to be classified as exposed to ICS during the entire follow-up, even during the follow-up time before their first ICS prescription [7]. The time between cohort entry and the first ICS prescription is called "immortal" and the misclassification of this immortal and unexposed period as "exposed" will result in immortal time bias [34, 35]. Two of the studies that reported important reductions in lung cancer incidence with ICS used this approach and were thus affected by immortal time bias [7, 10]. One study that specifically avoided this bias by properly classifying exposure over time reported an elevated HR of lung cancer incidence with ICS (2.23, 95\% CI 1.31-3.79) [13].

Few of the observational studies included a latency period in the analysis. This can be problematic, since without such latency consideration, a study will classify an incident lung cancer as exposed to ICS even if it was diagnosed a very short time after the first ICS prescription, which can bias the effect estimate $[26,36]$. Indeed, our sensitivity analyses showed that the HR estimate of 1.01 (95\% CI 0.94-1.08) was increased to 1.22 (95\% CI 1.15-1.29) and 1.25 (95\% CI 1.16-1.34) when the 1-year latency period was not included. Finally, protopathic bias is likely in this context since early respiratory symptoms of a yet undiagnosed lung cancer can be treated with bronchodilators before the clinical diagnosis is posed [23]. However, few observational studies considered this source of bias. Our sensitivity analyses showed that this bias can affect the HR either way.

A strength of our observational study is the new-user design that identified new users of long-acting bronchodilators without ICS, which defines a clear cohort entry point. Moreover, our study was designed to specifically avoid several of the time-related biases affecting some of the previous studies, namely immortal time, latency time and protopathic biases. In addition, the use of the Cox proportional hazards model is noteworthy, as it takes into account changes in ICS use and lung cancer incidence over follow-up time. Indeed, using simple rates produced a crude rate ratio of 1.56 versus the crude HR of 1.02 (table 2). However, while the former assumes that the incidence rate of lung cancer is constant over follow-up time, the latter allows for the lung cancer incidence to increase with time, adjusting for increasing ICS use over time.

Our study is first limited by exposure measures based on dispensed prescriptions, which could introduce some misclassification as it is unclear if the medication was used and the uncertainty as to proper inhaler technique in a real world setting. Furthermore, the cohort may have included some patients with asthma [37]. However, this number is probably small because of a cut-off of age 50 years for treatment initiation and the cohort entry criterion based on initiating treatment exclusively with long-acting bronchodilators that do not include ICS, a treatment approach contraindicated for asthma. Nevertheless, residual confounding from unmeasured confounders cannot be ruled out, in particular the unavailability of smoking data along with the inclusion of some patients who develop asthma after the age of 50 years and the lack of identification of patients with emphysema. These unmeasured confounders and residual confounding from COPD severity may explain our dose-response findings of low doses of ICS associated with a lower incidence of lung cancer and higher doses with a higher incidence, although these could also be due to random fluctuation.

In view of the methodological limitations present in several observational studies and the absence of evidence from randomised safety trial data, it may be prudent to put some more thought into the proposition of a placebo-controlled randomised trial at this time [16]. Our observational study that accounted for the sources of bias present in other studies did not find an association between ICS use and lung cancer incidence. Several randomised trials have been conducted in COPD on the basis of observational studies, such as the studies reporting major reductions in all-cause mortality with ICS in COPD that were shown to be affected by immortal time bias [32, 38, 39]. These large beneficial effects could not be corroborated by the subsequent randomised trials [27, 40]. Moreover, a trial of ICS to prevent lung cancer in COPD may be practically unattainable, as it would require a very large number of patients with COPD who do not use ICS. Indeed, $>30000$ patients with COPD would be needed for such a trial to confirm the findings of the observational study. 
Overall, observational studies are certainly valuable to assess the effects of medications in the real-world clinical setting and to complement or form the basis for randomised trials. Our study did not find that ICS use reduces the incidence of lung cancer in patients with COPD. It appears that the previous studies that showed such a reduction were affected by time-related and some confounding biases that our study design was intended to avoid. Thus, for the question of ICS potentially preventing lung cancer, the proposition of a placebo-controlled randomised trial is still premature and would warrant some caution.

Conflict of interest: S. Suissa reports grants and personal fees for advisory board work and lectures from Boehringer Ingelheim and Novartis, personal fees for lectures from AstraZeneca, outside the submitted work. S. Dell'Aniello has nothing to disclose. A.V. Gonzalez has nothing to disclose. P. Ernst has nothing to disclose.

Support statement: This research was funded in part by grants from the Canadian Institutes of Health Research (CIHR) and the Canadian Foundation for Innovation (CFI). S. Suissa is the recipient of the Distinguished James McGill Professorship award.

\section{References}

1 Rabe KF, Hurd S, Anzueto A, et al. Global strategy for the diagnosis, management, and prevention of chronic obstructive pulmonary disease: GOLD executive summary. Am J Respir Crit Care Med 2007; 176: 532-555.

2 Lozano R, Naghavi M, Foreman K, et al. Global and regional mortality from 235 causes of death for 20 age groups in 1990 and 2010: a systematic analysis for the Global Burden of Disease Study 2010. Lancet 2012; 380: 2095-2128.

3 Cavaillès A, Brinchault-Rabin G, Dixmier A, et al. Comorbidities of COPD. Eur Respir Rev 2013; 22: 454-475.

4 Kiri VA, Soriano JB, Visick G, et al. Recent trends in lung cancer and its association with COPD: an analysis using the UK GP Research Database. Prim Care Respir J 2009; 19: 57-61.

5 de Torres J, Marín JM, Casanova C, et al. Lung cancer in patients with chronic obstructive pulmonary disease incidence and predicting factors. Am J Respir Crit Care Med 2011; 184: 913-919.

6 Kiri VA, Fabbri LM, Davis KJ, et al. Inhaled corticosteroids and risk of lung cancer among COPD patients who quit smoking. Respir Med 2009; 103: 85-90.

7 Raymakers AJN, Sadatsafavi M, Sin DD, et al. Inhaled corticosteroids and the risk of lung cancer in COPD: a population-based cohort study. Eur Respir J 2019; 53: 1801257.

8 Parimon T, Chien JW, Bryson CL, et al. Inhaled corticosteroids and risk of lung cancer among patients with chronic obstructive pulmonary disease. Am J Respir Crit Care Med 2007; 175: 712-719.

9 Lee C-H, Hyun MK, Jang EJ, et al. Inhaled corticosteroid use and risks of lung cancer and laryngeal cancer. Respir Med 2013; 107: 1222-1233.

10 Liu S-F, Kuo H-C, Lin M-C, et al. Inhaled corticosteroids have a protective effect against lung cancer in female patients with chronic obstructive pulmonary disease: a nationwide population-based cohort study. Oncotarget 2017; 8: 29711-29721.

11 Lee YM, Kim SJ, Lee JH, et al. Inhaled corticosteroids in COPD and the risk of lung cancer. Int J Cancer 2018; 143: 2311-2318.

12 Jian Z-H, Huang J-Y, Lin FC-F, et al. The use of corticosteroids in patients with COPD or asthma does not decrease lung squamous cell carcinoma. BMC Pulm Med 2015; 15: 154.

13 Kok VC, Horng J-T, Huang H-K, et al. Regular inhaled corticosteroids in adult-onset asthma and the risk for future cancer: a population-based cohort study with proper person-time analysis. Ther Clin Risk Manag 2015; 11: 489-499.

14 Sørli K, Thorvaldsen SM, Hatlen P. Use of inhaled corticosteroids and the risk of lung cancer, the HUNT study. Lung 2018; 196: 179-184.

15 Raymakers AJN, McCormick N, Marra CA, et al. Do inhaled corticosteroids protect against lung cancer in patients with COPD? A systematic review. Respirology 2017; 22: 61-70.

16 Seijo LM, Soriano JB, Peces-Barba G. New evidence on the chemoprevention of inhaled steroids and the risk of lung cancer in COPD. Eur Respir J 2019; 53: 1900717.

17 Ernst P, Saad N, Suissa S. Inhaled corticosteroids in COPD: the clinical evidence. Eur Respir J 2015; 45: 525-537.

18 Brisson C, Major D, Pelletier E. Évaluation de l'Exhaustivité du Fichier des Tumeurs du Québec. Institut National de Santé Publique du Québec 2003. www.inspq.qc.ca/sites/default/files/publications/217rapportexhaustiviteregistrecancers.pdf

19 Ernst P, Gonzalez AV, Brassard P, et al. Inhaled corticosteroid use in chronic obstructive pulmonary disease and the risk of hospitalization for pneumonia. Am J Respir Crit Care Med 2007; 176: 162-166.

20 Suissa S, Dell'Aniello S, Ernst P. Long-term natural history of chronic obstructive pulmonary disease: severe exacerbations and mortality. Thorax 2012; 67: 957-963.

21 Suissa S, Patenaude V, Lapi F, et al. Inhaled corticosteroids in COPD and the risk of serious pneumonia. Thorax 2013; 68: 1029-1036.

22 Gonzalez AV, Coulombe J, Ernst P, et al. Long-term use of inhaled corticosteroids in COPD and the risk of fracture. Chest 2018; 153: 321-328.

23 Horwitz RI, Feinstein AR. The problem of "protopathic bias" in case-control studies. Am J Med 1980; 68: 255-258.

24 Lougheed MD, Lemiere C, Ducharme FM, et al. Canadian Thoracic Society 2012 guideline update: diagnosis and management of asthma in preschoolers, children and adults. Can Respir J 2012; 19: 127-164.

25 Suissa S. Immortal time bias in pharmacoepidemiology. Am J Epidemiol 2008; 167: 492-499.

26 Rothman K. Induction and latent periods. Am J Epidemiol 1981; 114: 253-259.

27 Calverley PM, Anderson JA, Celli B, et al. Salmeterol and fluticasone propionate and survival in chronic obstructive pulmonary disease. N Engl J Med 2007; 356: 775-789. 
The Lung Health Study Research Group, Wise R, Connett J, et al. Effect of inhaled triamcinolone on the decline in pulmonary function in chronic obstructive pulmonary disease. N Engl J Med 2000; 343: 1902-1909.

29 Pauwels RA, Löfdahl CG, Laitinen LA, et al. Long-term treatment with inhaled budesonide in persons with mild chronic obstructive pulmonary disease who continue smoking. European Respiratory Society Study on Chronic Obstructive Pulmonary Disease. N Engl J Med 1999; 340: 1948-1953.

30 Tashkin DP, Rennard SI, Martin P, et al. Efficacy and safety of budesonide and formoterol in one pressurized metered-dose inhaler in patients with moderate to very severe chronic obstructive pulmonary disease: results of a 6-month randomized clinical trial. Drugs 2008; 68: 1975-2000.

31 Vestbo J, Leather D, Diar BN, et al. Effectiveness of fluticasone furoate-vilanterol for COPD in clinical practice. N Engl J Med 2016; 375: 1253-1260.

32 Suissa S, Ernst P. Observational studies of inhaled corticosteroid effectiveness in COPD: lessons learned. Chest 2018; 154: 257-265.

33 Smith BM, Pinto L, Ezer N, et al. Emphysema detected on computed tomography and risk of lung cancer: a systematic review and meta-analysis. Lung Cancer 2012; 77: 58-63.

34 Suissa S. Effectiveness of inhaled corticosteroids in chronic obstructive pulmonary disease: immortal time bias in observational studies. Am J Respir Crit Care Med 2003; 168: 49-53.

35 Suissa S. Immortal time bias in observational studies of drug effects. Pharmacoepidemiol Drug Saf 2007; 16: 241-249.

36 Rivera DR, McGlynn KA, Freedman AN. Connections between pharmacoepidemiology and cancer biology: designing biologically relevant studies of cancer outcomes. Ann Epidemiol 2016; 26: 741-745.

37 Stupka E, deShazo R. Asthma in seniors: part 1. Evidence for underdiagnosis, undertreatment, and increasing morbidity and mortality. Am J Med 2009; 122: 6-11.

38 Sin DD, Tu JV. Inhaled corticosteroids and the risk of mortality and readmission in elderly patients with chronic obstructive pulmonary disease. Am J Respir Crit Care Med 2001; 164: 580-584.

39 Soriano JB, Vestbo J, Pride NB, et al. Survival in COPD patients after regular use of fluticasone propionate and salmeterol in general practice. Eur Respir J 2002; 20: 819-825.

40 Vestbo J, Anderson JA, Brook RD, et al. Fluticasone furoate and vilanterol and survival in chronic obstructive pulmonary disease with heightened cardiovascular risk (SUMMIT): a double-blind randomised controlled trial. Lancet 2016; 387: 1817-1826. 